\title{
La dot dagara : Les implications herméneutiques et intermédiales
}

\author{
Thadée Balouhib Somda KPANYAWNE ${ }^{1}$
}

\begin{abstract}
Some Africans more specifically some dagara, no more want nowadays that their future husbands pay the traditional dowry. Their parents think the contrary. The absence of these social principles deprives this human association of its title of sacred union. Certain antagonisms of the two different worlds appear of which one is inclined to westernization and the other to africanization. This tensive atmosphere led us to the reflection on 'The dagara dowry'. The problem that it creates is expressed by these terms: be known, explained, well interpreted to the service of the two rival parties. That is the reason of the following sub-titles:"The hermeneutic and intermedial implications". Our exegetics is based on the behavioral semiotic of the signs and the dagara oral discourses.
\end{abstract}

Key words: intermedial; hermeneutic; oral-discourses; semiotic; behavioral DOI: $10.24818 / \mathrm{DLG} / 2021 / 38 / 15$

\section{Introduction}

$\mathrm{L}$ a colonisation occidentale est, en toute apparence, boutée hors des frontières de l'Afrique. Cependant elle semble toujours prendre en tenailles les Africains par certains textes administratifs et lois qu'elle leur a laissés. Des dirigeants audacieux aux militaires révolutionnaires qui ont manifesté l'intention de revoir ces textes et lois, les ont payés cher; pour beaucoup c'est au prix de leur vie. Par conséquent certaines règles administrant des Africains, sont en déphasage avec des réalités, voire us et coutumes de ceux-ci. Si certaines de ces mœurs qui ont connu des temps immémoriaux, tiennent toujours bon, c'est qu'elles ont des valeurs qu'il faut reconnaître. Elles résolvent sûrement des problèmes sociaux réels au point que leur absence peut être suicidaire.

1 Thadée Balouhib Somda Kpanyawne, Université Joseph KI-ZERBO (UJKZ), Laboratoire des langues, discours et pratiques artistiques (LADIPA), Ecole doctorale lettres, sciences humaines et communication (LESHCO), Burkina Faso, thaddee_somda@yahoo.fr 
C'est le cas de certaines Africaines plus précisément des Dagara, qui veulent de nos jours, qu'à leurs mariages, aucune dot traditionnelle ne soit remise par leurs soupirants. Elles sont fortes de certains discours et textes administratifs de l'Etat burkinabè en cours. C'est donc plus fort que leurs parents qui pensent le contraire. Pour ceux-ci un vaste ensemble de valeurs individuelles, familiales, communautaires, ancestrales, qui diffèrent certes d'une société à une autre, accompagnent toujours la désignation mariage.

L'absence de ces principes sociaux ôte à ce genre de croisement humain le titre d'union sacrée qui n'en est pas une à leurs yeux. Apparaît alors un certain antagonisme de deux mondes différents dont l'un est enclin à l'occidentalisation et l'autre à l'africanisation. Cette atmosphère tensive a suscité en nous la réflexion sur La dot dagara. Le problème que celle-ci rencontre s'exprime en ces termes : le rejet parce que la dot est mal connue, mal expliquée, non interprétée au service des deux partis antagonistes. C'est la raison d'être du sous-titre suivant: "Les implications herméneutiques et intermédiales». Nous pensons que par la présente réflexion, la dot dagara sera bien connue, clairement expliquée et interprétée pour les deux partis opposés.

Notre exégèse s'orientera vers la sémiotique comportementale bâtie sur les signes et discours oraux dagara. Notre public cible sera l'Afrique traditionnelle en générale, le sud-ouest du Burkina Faso en particulier, plus précisément la communauté dagara fortement scolarisée. Dans le domaine matrimonial dagara, les signes et notamment les textes oraux qui y sont déployés, relèvent des discours dont la part artistique est notoire. A leurs aspects esthétiques nous grefferons notre regard herméneutique avec pour objectif que cette pratique sociale soit mieux comprise et acceptée par les deux mondes antagonistes. Tout l'espoir est que cette acception ne soit pas théorique mais pratique ou traduite dans les comportements des uns et des autres. Tout développement individuel et collectif, tout épanouissement humain passe par là c'est-à-dire par les conceptions sociales incluses dans les comportements des membres.

Notre approche méthodologique consistera à faire connaître le discours matrimonial dagara par un récit historique. Celui-ci sera perçu comme un signe sémiotique qui a son contenu. Nous en révèlerons la teneur par un travail herméneutique. C'est la raison pour laquelle le plan $\mathrm{s}^{\prime}$ initie par une approche théorique des choses et des Etres de sens. Ensuite nous nous intéresserons au plan de l'expression du récit dotal. C'est par ce biais que nous pensons accéder au plan de contenu de façon herméneutique. 


\section{Approches théoriques des choses et des êtres de sens}

La sémiotique est la science des signes et des systèmes de signification. Elle a permis d'élaborer des théories qui, en réalité, sont des méthodes d'approche des choses et des Etres considérés comme des signes et des systèmes de signification. Au nombre de ces théories voire méthodes, Terres de sens de J. Fontanille et N. Couegnas (2018). Cette œuvre théorique constituera la méthode primordiale de notre réflexion sur la dot dagara. Elle sera sous-tendue par d'autres : Formes de vie de J. Fontanille (2015), «Pour une sémiotique à base herméneutique» de J. Paré (1998), Du texte à l'action de P. Ricœur (1986). Avant tout n'oublions pas les études spécifiquement dagara véhiculant les notions théoriques propres à la dot de cette société.

\subsection{Définitions et notions sur la dot}

La dot relève des traditions, des mœurs, des coutumes, des habitudes de vivre. C'est pourquoi il importe de passer au peigne fin la définition de ces termes dont celui de dot est tributaire.

En premier lieu, le terme «coutume» est défini par certains dictionnaires comme la "Façon de vivre héritée du passé (particulière à un peuple ou à un groupe social)... Manière habituelle de se comporter. Synonyme: tradition» (Encarta ${ }^{\circledR}$ 2009. (C) 1993-2008 Microsoft du 04/05/2021). Etymologiquement, le mot «coutume » est paru à la fin du XIe siècle. Il a été créé à partir du mot latin «custume» provenant lui-même du Latin populaire "cosetudine», car le mot latin classique est "consuetudo, inis ». Celui-ci est le supin de "consuescere» composé de «con- (cum)» et de «suescere» qui signifie (vivre avec), «habituer» (www.lerobert.com du 04/05/2021).

En deuxième lieu, le terme «traditions" aurait paru dans les années 1291 de l'étymologie latine «traditio » provenant de «traditum». Ce dernier mot est le supin de "tradere » qui signifie "remettre, transmettre " (www.lerobert.com du04/05/2021). Le terme tradition était donc né dans le domaine juridique et signifiait "Remise matérielle d'une chose mobilière en vue soit d'en transférer la propriété, soit d'exécuter une obligation de délivrance». Du domaine juridique, le mot tradition est passée en croyance ou religion dans les années 1488 pour prendre le sens suivant: "Doctrine ou pratique, religieuse ou morale, transmise de siècle en siècle, originellement par la parole ou l'exemple (mais pouvant par la suite être consignée dans un texte écrit)». En 
1701 le vocabulaire tradition est en vogue dans les domaines de la connaissance, des techniques, des arts, des mœurs... et prend le sens suivant: "Manière, ou ensemble de manières, de penser, de faire ou d'agir, qui est un héritage du passé. " Il devient synonyme de "Coutume, habitude " (www.lerobert.com du04/05/2021).

En troisième lieu, le terme «mœurs" est défini par certains dictionnaires comme suit: étymologiquement, il provient du Latin «mores » qui est le pluriel de «mos, moris ». Déjà vers 1112 il se prononce «murs». Vers 1155 sa prononciation est «mors». En 1283 il se prononce "meurs» sans le « $\mathbf{o}$ ». Enfin son orthographe actuelle daterait de vers le $\mathrm{XIV}^{\mathrm{e}}$ siècle. Sa signification est: "Habitudes de vie, coutumes d'un peuple, d'une société ». Dans ce cas, «mœurs » se présente comme étant synonyme de «Coutume(s), usage(s)» (www.lerobert.com du04/05/2021).

Que retenir de toutes ces définitions ? Ce qui est frappant, c'est leur synonymie. Les termes traditions, mours, coutumes, habitudes de vivres, sont souvent pris pour des synonymes par les documents que nous avons consultés.

En conséquence nous ne sommes pas contre les conceptions anthropocentriques selon lesquelles certaines notions telles les us et coutumes, les traditions... sont propres à l'homme. Cependant tout Etre semble avoir ses habitudes de vivre appelées traditions, mœurs ou coutumes.

Pourquoi alors pousser certaines sociétés à renier leurs propres coutumes ? En effet c'est un constat : certaines sociétés - les plus rusées ou les plus fortes - conduisent insidieusement les autres à se haïr elles-mêmes en haïssant leurs coutumes. C'est dans ce sens que certains dagara, poussés dans un véritable embarras, ne savent plus s'il faut continuer d'aimer et pratiquer leurs propres cultures. Or mêmes les animaux ont leurs mœurs. Tout homme doit donc avoir et vivre ses mœurs d'où l'importance $\mathrm{du}$ thème suivant: $\mathrm{La}$ dot dagara: Les implications herméneutiques et intermédiales.

Après les définitions et les notions sur la dot, recourons aux notions sémiotiques pour l'étude de cette pratique sociale. 


\subsection{Notions sémiotiques}

Les notions sémiotiques visent à faire la synthèse des travaux de certains prédécesseurs comme Fontanille, Couegnas, Paré et Ricœur.

\subsubsection{Terres de sens de Fontanille et Couegnas (2018)}

Par Terres de sens, Fontanille et Couegnas visent des objectifs: d'abord montrer que nous ne vivons pas dans un seul monde mais dans des mondes qui sont, en réalité, des systèmes de signification. Nous sommes donc permanemment plongés dans des mondes voire des "terres de sens ». Ensuite cette œuvre montre comment accéder à ces significations. C'est en cela qu'elle est une méthode anthroposémiotique à laquelle nous aimerions recourir pour mener à bien notre réflexion par les aspects suivants de sa procédure : premièrement l'étude de terrain (J. Fontanille, N. Couegnas, $2018: 85-222$ ) dont le territoire français précisément rural du Limousin, est l'espace de prédilection. Le terrain a permis à Terres de sens d'être une méthode plus pratique que théorique.

Deuxièmement la recherche dans Terres de sens présente les imaginaires paysans et les utopies ouvrières à l'aide d'études antérieures (J. Fontanille, N. Couegnas, 2018 : 265-267). Dans cet ordre d'idée, légendes et rites ont retenu notre attention. Troisièmement la recherche dans Terres de sens présente des coopératives et autres formes de l'économie sociale et solidaire. Ce sont des formes de vie dont certaines caractéristiques sont "Autonomie et indépendance », "Education, formation et information » (J. Fontanille, N. Couegnas, $2018: 161) \ldots$

Terres de sens entretient des liens avec les travaux d'autres auteurs tels le co-auteur J. Fontanille, J. Paré et P. Ricœur.

\subsubsection{Fontanille, Paré et Ricœur}

Les liens que Terres de sens entretiennent avec les travaux d'autres auteurs sont des traits fondamentaux pour notre réflexion. Il n'est pas impossible que ces prédécesseurs aient influé sur l'œuvre principale de nos références.

Nous pensons notamment à J. Fontanille qui est co-auteur de cet essai de méthode et dont nous retrouvons la notion de "formes de vie " développée dans sa théorie intitulée effectivement Formes de vie et publiée en 2015. A ce sujet, Terres de sens véhicule ceci :

"Cette expérience de pensée a pour effet une transition de formes de vie.

La révolution critique portée par le mouvement anthropophage comporte 
en effet deux propriétés caractéristiques des formes de vie [...] Toute forme de vie s'affirme dans la congruence entre des systèmes de valeurs, des régimes de sens, des styles de comportements, etc.» (J. Fontanille, N. Couegnas, $2018: 40-42$ ).

Pour avoir eu souvent recours à cette œuvre, Formes de vie (J. Fontanille, 2015), nous savons que les coopératives dont il est question dans Terres de sens, sont des «formes de vie ». Il en est de même pour notre recherche : la dot dagara est une forme de vie que nous détaillerons infra.

Dans un article intitulé "Pour une sémiotique à base herméneutique», J. Paré circonscrit le problème suivant : la sémiotique était une grande mode dans les années 80. Mais elle "semble aujourd'hui dans le creux de la vague " à tel point que "Le chercheur qui inscrit ses travaux dans le domaine de la sémiotique littéraire, se pose de plus en plus de questions autant sur l'objet que sur la finalité de ses investigations »(J. Paré, 1998: 77-78). L'auteur, en engageant une telle réflexion, vise un objectif : examiner les raisons de cet épuisement et "indiquer des pistes qui permettraient au sémioticien d'entreprendre une tâche de redéploiement». L'une de ces pistes semble être l'option de Paré «Pour une sémiotique à base herméneutique (J. Paré, 1998 : 77-78). La sémiotique ainsi perçue intéresse notre réflexion par sa force interprétative que nous avons relevée comme un des traits caractéristiques dans Terres de sens de J. Fontanille et N. Couegnas (2018).

Nous recourons aussi à P. Ricœur (1986) qui partage cette idée de la sémiotique abondant dans le sens herméneutique ou interprétatif. Dans son œuvre Du texte à l'action, il précise davantage son intention par le soustitre « Essais d'herméneutique ». Pour lui :

"interpréter, c'est prendre le chemin de pensée ouvert par le texte, se mettre en route vers l'orient $d u$ texte... C'est pourquoi l'interprétation se confond avec la dimension sémantique de la parole elle-même: l'interprétation, c'est le discours lui-même, c'est tout discours. Néanmoins, je retiens d'Aristote l'idée que l'interprétation est interprétation par le langage avant d'être interprétation sur le langage » (P. Ricœur, 1986 : 156-157).

Après avoir fait le tour de ces théories essayons de voir comment celles-ci peuvent nous aider à mieux comprendre et le contenu de la dot et sa pratique. Et pour ce, intéressons-nous au plan de manifestation de cette mode sociale. 


\section{Plan de manifestation : Historique et mythologie}

Le plan de l'expression de la dot dagara reposera sur le récit de la dot et sur les mythes de cette pratique qui n'est pas sans épreuve en tant que forme de vie.

\subsection{Récit de la dot dagara ${ }^{2}$}

D'après nos informateurs, depuis le temps de nos ancêtres, lorsqu'un homme dagara prend la fille d'une famille pour en faire une femme, il doit accomplir un devoir que nous appelons la remise de la dot (Tur a caro $\mathrm{n} \iota$ a $\mathrm{d} \varepsilon \varepsilon)^{3}$. L'accomplissement de cette tâche sociale peut s'effectuer en plusieurs étapes. Celles-ci sont plus détaillées dans les œuvres suivantes: La dot et son rôle dans le mariage traditionnel (D. D. R. Kusiélé, 1999), Enquête sur les alliances dans la culture africaine notamment au pays dagari (sœur S. KP. D. B. Bèkuonè, 1994), Bu-nyèri ni bu-bè-nyèri (LE VISIBLE ET L'INVISIBLE) : Une interprétation de la mentalité animiste en guise de contribution à l'entreprise philosophique universelle (D. C. GBaanè, 1978) et Nisaal : L'homme comme relation, Tome I (D. C. GBaanè, 1983). Les détails qu'offrent ces œuvres sont très importants. Mais pour éviter leur répétitions inutiles, synthétisons-les en deux grands ensembles avec l'objectif suivant : c'est parce que quelque chose est due, est acquisitive, et qu'elle est appelée « dot» que la problématique est posée dès le début de notre réflexion.

\subsubsection{Premier groupe : Les rituels immuables}

Le premier grand ensemble s'appelle «Carv». Pour le différencier de l'autre qui est son homonyme homographe, on ajoute «kpëë » et cela donne l'expression "Car kpëë». Elle est longue. C'est pourquoi elle est

${ }^{2}$ Le vrai auteur de toutes ces histoires est la société dagara. Nous-même les connaissons mais par la recherche elles nous sont rappelées par nos informateurs comme Kpanyawnè Somda Kuu-yaa-san dit Gbèdaa et alii, département de Dissihn, village de Kognègan, le 23/03/2017.

${ }^{3}$ La traduction est phonétique, celle de l'API (Alphabet Phonétique International) avec conservation de certaines anciennes lettres parce que toujours plus usitées que les nouvelles. Voici quelques exemples où les premières lettres sont celles qui sont nouvelles et les autres sont les anciennes lettres, celles susceptibles $d^{\prime}$ être présentes dans le texte $: \hat{h}={ }^{\prime} h, f=g y, d={ }^{\prime} l,\left[\right.$ ou $B={ }^{\prime} m, n m=m w, y=n g$, $\mathrm{n}=$ ny. 
apocopée «Carv». Littéralement traduit, «Car kpëë» c'est le « grand Carv». Au plan littéraire c'est «Car kpëë» qui est le plus important. «Car kpëë » est «la vraie dot» ou «l'essence de la dot» ou aussi «la base de la dot» ou encore «le fond de la dot»... Elle est immuable, figée. Quelle que soit la fille, cette essence ne change pas et ce, sans distinction de famille, de village, de pays, de race, de religion, d'où notre titre : les rituels immuables.

D'aucuns voient en ce premier grand ensemble des sous-ensembles dont le premier est «Libir kpëë» souvent éponyme de tout le premier grand ensemble pour le différencier du deuxième. Littéralement traduit «Libir kpëë » est «l'argent grand ». Au plan littéraire c'est «l'essence de l'essence ». «Libir kpëë» ou «l'essence de l'essence» vaut trois cent soixante (360) cauris. En Dagara c'est «Kobr-a-ta li-zaa-ta». Cette somme n'est pas monnayable.

Le deuxième sous-ensemble comprend deux éléments réunis en un et indiqués par une seule locution : «Kob-tur-dicĩ-kobr-a-nuu» traduisible par cinq mille (5000) cauris pour soutien aux travaux champêtres et deux mille cinq cent (2500) cauris pour la part du berger. Ces deux éléments de ce deuxième sous-ensemble sont monnayables respectivement à cinq mille Francs CFA (5 000 F CFA) et à deux mille cinq cent Francs CFA (2 500 F CFA) soit le tout à sept mille cinq cent Francs CFA (7500 F CFA). Ils sont monnayables mais immuables, figés. Quelle que soit la fille, cette essence ne change pas et ce, sans distinction de famille, de village, de pays, de race, de religion, d'où notre titre : les rituels immuables.

\subsubsection{Deuxième groupe : Les rituels variables}

Le deuxième grand ensemble est connu sous l'appellation "Carv» aussi. Mais pour le distinguer du premier, on prend soin de tout dire de la

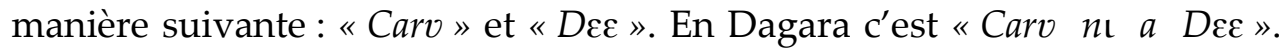
C'est donc le contexte circonscrit ou l'ajout de précision qui détermine le sens de «Carv». «Carv» et «Deq» sont alors les éléments constitutifs de ce deuxième grand ensemble. Ils sont des constituants rituels instables. Ils changent en fonction de certaines circonstances : lire infra.

Ici le premier sous-élément, "Carv», est un ensemble de cauris dont le nombre est toujours plus élevé que ceux du premier grand ensemble. Il est toujours et strictement égal mais jamais inférieur ou supérieur à celui qui avait été offert pour doter la mère de la fille à marier. Nos informateurs disent avoir été témoins des sommes suivantes offertes 
pour dot : dix mille (10 000) à trente mille (30 000) cauris. En Dagara : libipla tur-pié tı tân libi-pla Tur-lızer-nt-pié. Ces cauris sont monnayables.

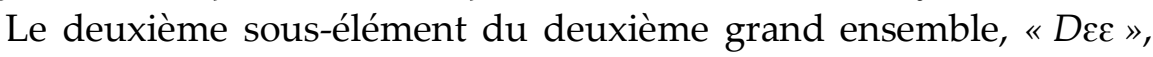
est constitué d'animaux dont les espèces et le nombre sont toujours et strictement égaux mais jamais inférieurs ou supérieurs à ceux qui avaient été offerts pour doter la mère de la fille concernée. En général c'est entre deux et trois veaux auxquels l'on joint des poules, des pintades... Ces animaux sont monnayables à moindre frais soit vingt-cinq mille Francs CFA (25000 F CFA) pour la génisse et vingt mille Francs CFA (20 $000 \mathrm{~F}$ CFA) pour le veau soit quarante-cinq mille Francs CFA (45 000 F CFA) pour obtenir les deux veaux.

La dot dagara qui est une "forme de vie» selon les termes de J. Fontanille (2015) tient de certains imaginaires similaires à ceux évoqués dans Terres de sens (J. Fontanille, N. Couegnas, 2018 : 265-267). Considérons alors certains mythes dagara sur la dot. Ils peuvent éclairer les esprits et guider les comportements à adopter à l'endroit de cette pratique.

\subsection{Mythologie}

La dot tient de la mythologie c'est-à-dire une histoire qui n'est pas vraie mais qui peut l'être et dont le plus important est surtout l'objectif visé : son but est d'expliquer la raison d'être d'une réalité. Et la plupart des réalités africaines en général et dagara en particulier, tiennent d'un mythe. En guise d'exemples : le mythe dagara de la création pour expliquer pourquoi la souffrance et la mort sur terre; le mythe dagara pour expliquer pourquoi le cochon a interdit sa descendance de marier celle des chèvres.

\subsubsection{Mythologie $\mathrm{I}^{4}$}

Il était une fois l'ancêtre dagara constata ceci : les hommes les plus forts avaient toutes les femmes de la société à leur disposition tandis que les plus faibles n'avaient rien. Pire lorsqu'un plus faible prenait le risque d'aller vers une fille avec l'intention d'en faire sa propriété, un plus fort pouvait la lui interdire voire retirer tant que cela lui faisait plaisir. Voilà

\footnotetext{
${ }^{4}$ L'ordre est de nous et par importance que nous accordons aux mythes: le premier sert de base aux autres. Rappel des informateurs : Kpanyawnè Somda Kuu-yaa-san dit Gbèdaa et alii, département de Dissihn, village de Kognègan, le 23/03/2017.
} 
pourquoi l'ancêtre dagara institua le mariage. Et pour que chacun puisse avoir une femme, l'ancêtre dagara décida que toute la famille se batte pour protéger les femmes de ses fils.

\subsubsection{Mythologie II}

Il était une fois l'ancêtre dagara constata ceci : beaucoup de filles et fils dagara n'arrivaient pas à se contenter d'un seul partenaire et cela causait d'énormes difficultés à la société. En effet lorsqu'un fils dagara allait avec plusieurs femmes, il était automatiquement reconnu comme le géniteur de tous les enfants de cette dame. Mais lorsque la femme allait avec plusieurs hommes en même temps, chaque partenaire réclamait la paternité des enfants de cette dame. Très souvent cela se terminait par des disputes sanglantes voire mortelles. Voilà pourquoi l'ancêtre dagara institua d'abord le mariage. Ensuite il bâtit l'organisation sociale entière sur des «bouches». Littérairement traduit ce sont des principes ou aussi des règlements, ou encore des lois, en Dagara «N $\mathrm{N} \varepsilon »$. Le tout premier principe est : la femme, elle et elle seule, connaîtra et saura indiquer qui est le père de ses enfants. Ce fut depuis ce jour que la matronymie fut en premier lieu. Un autre règlement est : le père, lui et lui seul, connaîtra et saura indiquer qui est la mère de ses enfants. Depuis ce jour la patronymie fut. Un autre principe est : les hommes qui « pensent qu'ils peuvent $»^{5}$, en Dagara "A bcl nâa tcr a fâw $\hat{a} »$, pourront prendre plusieurs femmes. C'est depuis ce jour que les Dagara qui «pensent qu'ils peuvent», épousent plus d'une femme. Encore une autre loi: toute femme qui connaît un homme autre que son époux, doit le dire pour que la faute soit réparée. C'est depuis ces temps-là que l'adultère est prohibé au pays dagara.

\subsubsection{Mythologie III}

Il était une fois un homme avait une femme. Celle-ci le cocufiait. Tout le monde le savait. Mais, lui, n'en avait aucune preuve. En effet il ne les avait jamais pris la main dans le sac. Alors son chagrin ne faisait que $\mathrm{s}^{\prime}$ accroître de jour en jour. Un jour son père lui dit :

- Mon fils, le jour où ton beau-père nous remettait sa fille en mariage, te souviens-tu de ce qu'il avait dit ?

- Non! père, répondit le fils.

\footnotetext{
${ }^{5}$ Cette expression sera mieux comprise à la phase herméneutique.
} 
- Alors, reprit le géniteur, ton beau-père disait ceci : "Je ne vous donne pas ma fille! Je vous la prête pour qu'elle fasse des enfants pour votre famille». Et le père poursuivit: «voici trois cent soixante (360) cauris. Prends-les. Va, conte ton chagrin à ton beau-père et remets-lui ces cauris ». Le mari cocu fit ce que dit son père.

Le beau-père écouta d'abord l'histoire racontée au sujet de sa fille par le mari cocu. Il reçut ensuite les trois cent soixante (360) cauris. Puis il libéra son gendre. Aussi il appela sa fille pour entendre sa version. Mais la femme nia qu'elle ne trompait point son époux.

- Es-tu sûre de ce que tu dis ma fille, demanda une première fois le père?

- Père, je suis sûre de ce que je dis, répondit la fille.

Le père questionna une deuxième, une troisième fois sa fille. Et celle-ci donna la même réponse. Papa dit :

- C'est parce que tu es femme que je te poserai cette même question pour la quatrième et la d-e-r-n-i-è-r-e fois, en Dagara "A per baara na", avec insistance sur l'expression "dernière fois » : es-tu sûre de toi ma fille?

- Oui je suis sûre de moi père, répliqua la fille avec fermeté !

Alors le père présenta à sa fille les trois cent soixante (360) cauris et dit :

- Voici. Ces cauris m'ont été remis par ton mari. Mes frères, mes sœurs, toute ta famille et moi, nous ne les accepterons que si toi, tu les acceptes. Et si tu les acceptes, ces cauris feront de toi la femme de ton mari uniquement.

- Je les accepte père, dis la fille sans la moindre hésitation.

Cependant le père savait que sa fille mentait. Et pour l'effrayer, il dit : si tu acceptes les trois cent soixante (360) cauris, nous aussi nous les acceptons. C'est pourquoi si tu trompes encore ton mari, celui-ci mourra...

Et le père scruta sa fille espérant avoir de sa progéniture le moindre frissonnement d'angoisse. Mais sa fille resta imperturbable. Pour effrayer davantage sa fille, le père ajouta : Après la mort de ton mari, toi aussi tu mourras... Et le père scruta encore sa fille espérant avoir de sa progéniture le moindre frissonnement d'angoisse. Mais son enfant resta imperturbable. L'évocation de la mort de son époux et de sa propre mort n'eut pu l'émouvoir.

Après tout le père mourut sans remédier à sa malédiction. Cela se dit en Dagara «A sââ wa tı kpi $\varepsilon$ be ir a $v$ noor ber $\varepsilon$ ». 
Et un jour cette femme têtue cocufia encore son mari. Celui-ci, peu de temps après, mourut. Dès que la femme porta le "gânë ${ }^{6}$, elle tomba elle aussi raide morte.

C'est pourquoi le Dagara dit : «La 'hââ sa kõ 'hââa ce qui se traduit par « Il vaut mieux en rire que d'en pleurer».

\section{Plan de contenu : Herméneutique}

Le plan de contenu sera d'abord interprétatif en nous référant à celui (plan de contenu) de Terres de sens de J. Fontanille et N. Couegnas (2018). Les idées de Paré (1998 : 77-78) importent aussi : notre intention est "d'entreprendre une tâche de redéploiement » du discours oral sur la dot. Cette idée de la sémiotique perçue dans le sens herméneutique, interprétatif, $\mathrm{P}$. Ricœur (1986 : 156-157) la partage aussi : «interpréter, c'est prendre le chemin de pensée ouvert par le texte... l'interprétation est interprétation par le langage avant d'être interprétation sur le langage ». Le plan de contenu ouvrira ensuite les interprétations de la dot vers des horizons où des solutions sont envisageables.

\subsection{Interprétation : La dot dagara est pour protéger et non pour nuire à la fille}

La dot dagara, si elle est bien interprétée, comprise et acceptée, elle peut apparaître comme l'un des premiers ancêtres du 8 mars, Journée internationale de la Femme baptisée ainsi en l'année 1975 déclarée comme "année de la femme " par l'Organisation des Nations Unies (ONU). Savoir qu'au pays dagara, la dot n'est pas la seule institution sociale reconnaissant et valorisant ainsi la femme. En affirmant cela nous pensons à d'autres formes de vie sociale comme la matronymie exclusivement traitée dans certains de nos travaux en voie de publication. Gardons à vue que dès le début de ce paragraphe, nous avons formulé la condition si « la dot est bien interprétée ». Considérant donc cette expression, interprétons les mythes. Révélons leurs significations profondes pour une meilleure

\footnotetext{
${ }^{6}$ Le «gânë» est une sorte de corde. Elle ceint soit la taille de la veuve soit le poignet du veuf durant toutes les funérailles dagara. Pour les cartésiens, le "gânë » sert à retenir la veuve ou le veuf aux moments de difficultés voire de fuite et à éviter le pire. Pour les véritables significations dagara du port du "gânë», lire la suite.
} 
compréhension de la dot dagara dont l'acceptation doit être visible non par la théorie mais par les comportements qui prônent le développement ou l'épanouissement humain par les formes de vie sociale.

\subsubsection{Interprétation des mythes}

Tout aspect du rituel "invite à rechercher la signification de cette pratique à l'intérieur d'une forme de vie et d'un mode d'identification» (J. Fontanille et N. Couegnas, 2018: 38-40). Autrement dit il importe de toujours chercher à comprendre la signification de la dot avant son rejet et ce, à l'intérieur d'une forme de vie dagara et d'un mode d'identification de cette communauté. Reprenons certains passages des mythes dagara. Citons-les et à l'aide de nos informateurs et nos expériences personnelles, essayons de révéler leurs significations profondes.

La mythologie I exprime ceci : "Lorsqu'un plus faible prenait le risque d'aller vers une fille ». On n'a pas dit aller vers une femme. Cela veut dire que le mariage n'existait pas. Il n'y avait que des personnes féminines libres, des filles sans le titre de femme ou d'épouse. En effet ce titre "femme » indique qu'il existe un mari, un possesseur. Cela veut dire que la procréation était donc l'apanage des plus forts.

Selon la mythologie I, le mariage social prime sur le mariage individuel. Les signes indicateurs sont: "L'ancêtre dagara décida que toute la famille se batte pour protéger les femmes de ses fils ». Mais une question se pose : comme aujourd'hui, il n'existe plus de plus forts à cause de la loi qui protège tous les citoyens, est-ce que le mariage social a encore sa raison d'être? Oui, en ce sens que tous les problèmes du couple résolus collectivement réussissent mieux et toujours par rapport aux solutions individuelles qui ont moins de force de frappe. Ce mythe indique que la vraie femme dagara est aussi celle de tous les frères de l'époux au sens africain.

Il n'est point question ici d'intimité stricte au sens d'autres cultures qui ne souffrent pas d'entendre la moindre idée de partage de femme. Cela signifie que la femme est étrangère venue d'ailleurs mais acceptée, entretenue, protégée, émancipée par tous les autres membres de la famille chacun à son titre social. En aucune façon les deux conjoints ne peuvent décider de leur séparation ou divorce sans le consentement familial. Nos informateurs nous ont fournis beaucoup d'exemples de couples où le conjoint rejeté par l'autre est toujours recueilli par la famille qui responsabilise un ou plusieurs de ses membres pour lui venir en aide. 
Composant avec le temps et par certains stratagèmes, la famille finit par toujours réconcilier les conjoints qui étaient disjoints.

En somme on peut dire premièrement que le bon ou vrai mariage dagara à la manière ancestrale est indissoluble. On y reviendra infra par des acteurs appelés "bergers" et qui souffrent plus que les conjoints surtout au moment de donner la dot et dans les circonstances conflictuelles du couple. Le divorce est autorisé mais difficile à réaliser sauf cas de force majeur : infidélité, sorcellerie, vol, illégitimité pour les filles non dotées qui ne sont donc pas femmes par manque de dot... Deuxièmement c'est cette mythologie I qui est à la base des deux autres mythes sur la dot. Il est fondamental par l'institution du mariage. C'est le commencement de tout ce qui est en rapport avec la dot.

C'est pourquoi dans la mythologie II, il est aussi dit: "Voilà pourquoi l'ancêtre dagara institua d'abord le mariage. Ensuite il bâtit l'organisation sociale entière sur des «bouches»». Les termes méritant une attention particulière sont: "d'abord», "Ensuite», "entière » qui hiérarchisent les institutions. Le mariage vient en premier lieu suivi de l'organisation sociale. Celle-ci ne repose pas partiellement mais entièrement sur des principes, en Dagara «Nec», qui suivent, eux aussi, un plan hiérarchique.

Le tout premier et donc le plus important est : « la femme, elle et elle seule, connaîtra et saura indiquer qui est le père de ses enfants ». Ce principe premier se passe de commentaire tant sa signification est réelle à travers la femme qui, seule, connaît le père de ses enfants. C'est une vérité générale ! De nos jours la science peut passer outre la femme et par l'ADN pour connaître le père des enfants de celle-ci mais on note quelques inconvénients dont l'inaccessibilité de ces méthodes scientifiques, etc.

C'est toujours dans la mythologie II qu'il y a révélation de l'instauration de la matronymie: "Ce fut depuis ce jour que la matronymie fut». C'est le matriarcat. Ce système social ne semble pas être l'apanage des Dagara mais de l'Afrique entière d'après L'unité culturelle de l'Afrique noire de C. A. Diop (1982). Au jour d'aujourd'hui, tout dagara se définit d'abord par la patronymie. C'est l'effet de la colonisation. Sinon tous les Africains sans exception, selon C. A. Diop (1982), relèvent d'abord de la matronymie sans que celle-ci soit un fléau pour l'Etre masculin. L'homme et la femme africains étaient vraiment égaux c'est-à-dire que sans pratiquer les mêmes activités, chacun était le complément de l'autre. 
La patronymie dagara, telle qu'elle est pratiquée de nos jours, a une signification plus profonde. Elle révèle des Dagara plus colonisés que libres, plus néo-colonisés qu'affranchis. Paradoxe: pour s'affranchir réellement de la néo-colonisation, les Dagara doivent absolument retrouver leurs patronymes perdus. Ce faisant ils pourront continuer d'obéir aux injonctions administratives burkinabè qui sont coloniales et néocoloniales par le fait de soumettre les Dagara non pas à leur matriarcat ancestral mais au patriarcat qui relève purement et simplement des mœurs occidentales ou aryennes ${ }^{7}$ selon les termes de C. A. Diop (1982 : 141-142 ; 185).

Ce faisant aussi les Dagara pourront continuer de respecter leur matriarcat qu'ils veulent transformer en patriarcat par force pour obéir à l'administration burkinabè qui leur exige des patronymes qu'ils ont mais qui ne figurent pas sur leurs extraits d'acte de naissance à cause du colon qui a expressément fait une mauvaise transcription tant les intentions inhumaines et indicibles de celui-ci sont notoires.

Même si aujourd'hui, tout dagara, pour montrer qu'il accepte toujours la colonisation, se définit par le patriarcat, il importe de faire remarquer que $l^{\prime}$ Etre tout entier dagara commence d'abord par le matriarcat et le patriarcat ne vient qu'après. En effet, dès sa conception, l'Etre dagara a un matronyme, en Dagara «Belu ». Le patriarcat, en Dagara "Yir dowlo», ne vient qu'après naissance si et seulement si la mère reconnaît que cet enfant est réellement de telle patronymie.

Alors la mythologie II signifie, en réalité, que «la matronymie fut en premier lieu » pour laisser place au règlement deuxième qui est : "le père, lui et lui seul, connaîtra et saura indiquer qui est la mère de ses enfants ». Par ce principe «la patronymie fut». Elle vit le jour depuis ce temps. La mythologie II révèle aussi la signification profonde du principe selon lequel «les hommes qui "pensent qu'ils peuvent», en Dagara " $A$ bel nâ ter a fâw $\hat{a} »$, pourront prendre plusieurs femmes ».

L'expression «les hommes qui «pensent qu'ils peuvent» » est très significative dans cette société Dagara. Elle dit tout, tant le pouvoir physique que moral, social, économique, politique... Cela veut dire que $c^{\prime}$ est dur : qui s'y frotte s'y pique ; c'est très clair que la polygamie n'est pas

\footnotetext{
${ }^{7}$ Aryen : terme que Cheikh Anta DIOP utilise pour faire la différence avec la race noire qu'il appelle type méridional. Lire L'unité culturelle de l'Afrique noire de Cheikh Anta DIOP (1982 : 141-142 ; 185).
} 
pour tout le monde, ce n'est pas pour n'importe qui. Mais surtout cela veut dire que le polygame peut réussir par ce système tout comme il peut $\mathrm{y}$ laisser des plumes. C'est depuis ce jour que les Dagara qui «pensent qu'ils peuvent ", épousent plus d'une femme.

Une autre loi tirée de la mythologie II : " toute femme qui connaît un homme autre que son époux, doit le dire pour que la faute soit réparée. C'est depuis ces temps-là que l'adultère est prohibé au pays dagara ». Comprendre par-là que la femme fidèle est bénie. Celle infidèle est maudite. Cela n'est pas l'apanage du Dagara. En termes plus clairs, toute femme qui ne croit pas à ces institutions ancestrales, et tient à partager plusieurs partenaires tout en étant mariée, le fait à ses risques et périls...

Ici foisonnent beaucoup de non-dits. Le premier est qu'en cas d'adultère c'est l'homme qui meurt et non la femme. Voir par-là un véritable équilibre entre le plein droit octroyé à l'homme de prendre plusieurs épouses contre le plein droit de la femme de décider de la vie de son époux. Plusieurs femmes ce sont aussi plusieurs risques d'épouses adultères et donc de nombreux dangers de mort de l'époux polygame! Suite des non-dits : il ne revient pas à la femme mais à l'homme de prendre toutes ses précautions non seulement pour d'abord très bien traiter la femme et éviter ainsi sa mort prochaine. Ensuite il ne doit pas se tromper et manger la nourriture préparée des mains de sa femme infidèle. Puis ce sera de sa faute s'il accepte boire de l'eau ou se doucher avec de l'eau... toute chose remise à lui par sa femme dont il est mis au courant de son infidélité.

Toujours par des non-dits l'on sait que tous les époux dagara se prémunissent contre ces infidélités devenues très récurrentes de nos jours et que seuls les incrédules y succombent. Les femmes aussi se prémunissent. Mais alors où va-t-on... ?

Nous pouvons clore la mythologie II en insistant sur le fait que ses significations révélées constituent une suite logique de celles offertes par la mythologie I. Ses connotations peuvent aider à répondre au comment ou à la raison d'être de certaines institutions indispensables à la société dagara. On en dénombre au total quatre : la matronymie, la patronymie, la polygamie et l'adultère. Le sens de l'instauration de ce dernier principe est plus approfondi dans la mythologie suivante. C'est donc en fonction des implications entre ces mythes que nous les avons classés : que le précédent facilite la compréhension du suivant. 
Ouvrons l'analyse de la mythologie III par la parole du père géniteur de la femme adultère : "Je ne vous donne pas ma fille! Je vous la prête pour qu'elle fasse des enfants pour votre famille ». C'est l'origine de la loi ancestrale dagara selon laquelle aucun homme n'achète une fille dagara par le mariage encore moins par la dot qui n'en est qu'une composante (du mariage). Le mariage est un contrat social d'entente mutuelle qui s'exprime de la sorte : premièrement les parents de la fille prêtée au mari peuvent reprendre leur enfant à tout moment et sans explication aucune à personne. Et la famille alliée doit se rappeler constamment cette close.

Deuxièmement la raison fondamentale qui peut surtout pousser des parents à reprendre leur fille prêtée au mari est la maltraitance. De par le passé ce retour de la femme chez ses parents était fréquent et même très fréquent. C'était alors que l'on pouvait entendre ceci des géniteurs : "Si vous n'avez plus besoin de votre femme, nous, nous en avons besoin car c'est avant tout notre enfant! » Les enfants reviennent toujours de droit au mari par la dot, les trois cent soixante cauris. Cette somme remet de façon illocutoire les enfants à leur père tout comme la parole des parents prêtant leur fille au mari sacre éternellement cette même fille à ses géniteurs.

Partant tout dagara prend soin de sa femme, la mère de ses enfants. Et il est institué dans la société que c'est une honte de se laisser retirer sa femme par les parents de celle-ci surtout pour cause de maltraitance. L'expérience a montré que tous les Dagara privés de leurs femmes de cette manière, ont, par la suite, éprouvé de graves difficultés à épouser de nouveau une autre femme. En effet toutes les familles où ils vont en chercher, les indexent comme étant de mauvais "gestionnaires » de femmes.

Problèmes modernes importés ou provenant des mœurs d'ailleurs : avoir un mari de nos jours est non seulement difficile et complexe mais surtout, une fille dagara qui se laisse retirer aujourd'hui par ses parents, n'arrivera même pas à domicile parental qu'elle apprendra qu'une autre l'a remplacée. C'est révélateur de beaucoup de mauvais comportements modernes importés ou provenant des mœurs d'ailleurs : premièrement cela indique que les hommes n'aiment plus réellement leurs femmes ; à peine partie elle est aussitôt remplacée par une autre qui certainement subira aussi le même sort! Deuxièmement on note que des femmes acceptent prendre la place des autres femmes chassées par leurs maris; des femmes poussent même des hommes à chasser leurs épouses pour prendre leurs places : nous avons la preuve que c'est la femme qui fait le 
malheur de la femme oubliant que c'est le «Renard passe-passe... Chacun à son tour!».

Troisièmement, le mariage dagara, social, typiquement ancestral, vient à bout de tous ces problèmes modernes importés et c'est d'actualité. Ces problèmes modernes évoqués ne sont que le lot des Africains dits aussi modernes et qui ont tué le mariage social ou ancestral.

"Je ne vous donne pas ma fille! Je vous la prête pour qu'elle fasse des enfants pour votre famille » dit le père de la fille. C'est aussi l'origine de la loi ancestrale dagara selon laquelle on ne peut enterrer une femme d'origine dagara sans qu'aucun membre de la famille de celle-ci ne s'en vienne jusque dans la tombe ou dans la dernière demeure de celle-ci pour s'assurer de la bientraitance de sa parente. D'après nos informateurs : « Si vous enterrez une fille dagara sans prévenir ses parents ou sans aucun accord avec sa famille, celle-ci a tout droit, selon les coutumes, d'exiger son exhumation». N'est-ce pas une autre preuve parmi tant d'autres que la coutume dagara protège assez la femme?

Notons autres révélations profondes de la mythologie III : «Et le père poursuivit : voici trois cent soixante (360) cauris. Prends-les. Va, conte ton chagrin à ton beau-père et remets-lui ces cauris ». On pense y voir l'origine de la dot, les trois cent soixante (360) cauris ou «l'essence de l'essence », en Dagara «Libir kpëë ». Les autres cauris, les bœufs et autres animaux seront institués après, toujours sur la base de "Je vous prête ma fille », etc. Et, parce que cette fille est devenue femme d'une autre famille, elle ne peut plus travailler pour donner à manger à sa famille d'origine, pour aider celle-ci à élever les animaux, en somme pour enrichir les siens, c'est alors que les autres cauris, les bœufs et autres animaux ont été institués pour compensation mais très après.

Et le père poursuivit: "voici trois cent soixante (360) cauris. Prendsles. Va, conte ton chagrin à ton beau-père et remets-lui ces cauris ". Pourquoi aller voir le beau-père ? C'est parce qu'il est toujours « propriétaire » voire géniteur de sa fille et il a toujours son mot à dire. C'est surtout pour qu'il soit toujours responsable de sa fille qu'il a confiée aux autres et qui n'est pas de bonnes mœurs. Serait-il la source des comportements répréhensibles de sa fille? En tout cas celle-ci ne fait point honneur tant à sa famille qu'à l'éducation donnée par celle-ci à ses filles !

En conséquence "Le père appela sa fille pour entendre sa version. Mais la femme nia»: ici il y a mensonge. Et au fur et à mesure, la menteuse devient têtue. Elle s'obstine même. Entendre par-là une signification plus 
profonde : dans la société dagara, la mort d'une femme adultère, est donc due à un mensonge, à une obstination et non à la malédiction du père ou à quiconque! La femme adultère est seule responsable !

Le passage "Le père présenta les cauris à sa fille» semble révéler l'origine du fait qu'il faut toujours présenter ces cauris à la fille ou à la future femme. Cela vaut-il la peine dans toutes les situations? Cette question vaut par le passage suivant: "Cependant le père savait que sa fille mentait... ». Jusqu'à ce niveau de conversation entre père et fille, il y a toujours un problème visible à travers le manque de confiance entre les deux interlocuteurs. Ce problème est que la fille est devenue têtue.

En réalité le père vise à éprouver sa fille en l'effrayant: «Et pour l'effrayer, il dit: si tu acceptes les trois cent soixante (360) cauris, nous aussi nous les acceptions. C'est pourquoi si tu trompes encore ton mari, celui-ci mourra». Pourquoi le père évoque la mort? Dans l'ancien temps, c'était ainsi que les choses se passaient dans la société dagara : soit Dieu, donc tout était sacré, soit la mort. Pourquoi commencer par la mort du mari ? C'est juste pour voir si sa fille l'aimait réellement car le conjoint c'est l'amour, c'est tout le cœur de l'autre conjoint! Pourquoi terminer par la mort de la femme : là aussi c'était pour voir si elle-même s'aimait ou avait pitié d'elle-même !

Dans tout cela l'attitude du père est révélatrice tant d'amour paternel, familial que d'espoir et d'angoisse qu'il vit lui-même en lieu et place de sa fille. C'est mitigé : "Et le père scruta sa fille espérant avoir de sa progéniture le moindre frissonnement d'angoisse. Mais sa fille resta imperturbable ». Le problème de manque de confiance va crescendo en ce sens que la fille est devenue têtue: "Le père ajouta: Et après, toi aussi tu mourras... Et le père scruta encore sa fille espérant avoir de sa progéniture le moindre frissonnement d'angoisse. Mais son enfant resta imperturbable». Le problème de manque de confiance prend un degré de plus dans le sens péjoratif. La fille est réellement têtue. Toute la mythologie III se résume en un problème de manque de confiance.

Les conséquences sont: "Après tout le père mourut sans remédier à sa malédiction ». Ce passage renferme beaucoup de non-dits. Est-ce par oubli que le père ne remédia pas à sa malédiction? Si oui c'est un acte inconscient! Le père l'a-t-il sciemment fait ? Si oui pourquoi ? Mais après analyse approfondie, toute question peut trouver sa réponse dans le manque de confiance exprimée ci-dessus. La confiance perdue peut être source de tout sauf le bien. Cependant ce mythe III vise à indiquer l'origine du fait que la femme est condamnée à être fidèle à son époux. 
En effet on a visiblement la preuve que la malédiction du père visait à effrayer sa fille ou à la pousser par tous les moyens pour qu'elle se confiât à lui. Par ce mythe, on peut entrevoir aussi l'origine de la nature complexe de la femme tant et si bien que même son père ne peut plus lui faire confiance. C'est le mythe de la femme capable des choses impensables, indicibles... Elle est capable de tout: la mort et même sa propre mort.

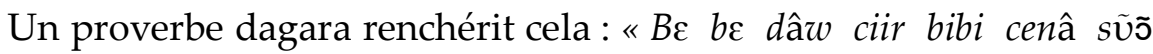
nè », ce qui se traduit par "Tout enfant sachant marcher ne peut être exempt d'accusation de sorcellerie ». Celui-ci a coutume, à l'aide de ces petits pas, de faire un détour quelque part où il renverse tout, fait du beau gâchis à l'insu de tous. Puis il revient s'asseoir tout innocent. A la question : qui a fait ce gâchis? Alors tout le monde s'accuse sauf l'innocent qui ne sait ni ne peut dire ce qu'il a fait.

Cette mythologie III peut aussi indiquer l'origine du «gânë»: pendant les funérailles la femme «doit porter le "gânë». Pourquoi ? Voici les sens réels $d u$ port $d u$ "gânë »: pour l'esprit cartésien qui tend à prendre le dessus or il est des moindres, le port du « gânë » est pour retenir la veuve aux moments opportuns et éviter ainsi qu'elle s'échappe car le pire peut vite arriver de cette fuite. Mais l'une des toutes premières significations du port du « gânë » est ceci : l'homme a perdu la valeur de sa fidélité par tous les moyens. La femme, elle, a conservé les valeurs de sa fidélité par tous les moyens dont le «gânë ». Le port de cet objet est pour montrer au vu et au su de tous, la fidélité de la femme estimée au-dessus de celle de l'homme.

Une autre des toutes premières significations du port du «gânë » est la légitimité. Une femme qui n'est pas dotée n'est pas légitime. Elle ne peut, en aucune façon, porter le "gânë ». La femme dotée à qui l'on refuse le "gânë », a tout droit de réclamer sa légitimité. Si on le lui refuse sans raison valable, cela devient un problème familial, de la saleté qui entache la famille entière. En Dagara cela se dit «Bawr a » ou «Dewr a ». La colère des ancêtres familiaux peut sévir.

On voit bien que la dot n'est pas contre la fille dagara. Elle la protège par tous les moyens et contre tout. Trois vertus sont à retenir $\mathrm{du}$ "gânë »: la légitimité, la fidélité et la prévention contre le pire ou le suicide. Elles (ces trois vertus) tiennent du seul pouvoir illocutoire de la dot mais surtout des trois cent soixante (360) cauris ou "l'essence de 
l'essence », en Dagara «Libir kpëë ». L'origine de ce pouvoir est lointaine, complexe mais déjà expliquée en ses étapes suivantes :

La première se situe au niveau du père remettant les trois cent soixante (360) cauris à son fils cocu. La deuxième étape présente le mari cocu recevant de son père les trois cent soixante (360) cauris. C'est le père qui fournit ces cauris et non l'époux de la femme. La troisième étape indique le mari cocu transmettant les trois cent soixante (360) cauris à son beau-père. La quatrième étape montre le beau-père recevant de son gendre les trois cent soixante (360). La sixième étape se situe toujours au niveau du beau-père remettant les trois cent soixante (360) cauris à sa fille. La septième étape présente la fille acceptant de son père les trois cent soixante (360) cauris.

La huitième étape indique le témoignage de toute la famille de la fille ; tous les siens de celle-ci, pour montrer qu'ils ne sauront aller contre sa volonté, acceptent les trois cent soixante (360) cauris. La neuvième étape montre le père présentant les conséquences de leurs acceptations ou de leurs paroles données en deux phases : "ton mari... mourra»; "Après la mort de ton mari, toi aussi tu mourras... ». La dixième et dernière étape présente l'effet illocutoire des trois cent soixante (360) cauris en deux phases aussi : le mari cocu, "peu de temps après, mourut»; "Dès que la femme porta le "gânë », elle tomba elle aussi raide morte ».

La morale de la mythologie III a un sens profond : "C'est pourquoi le Dagara dit: "La 'hââ sa kõ 'hââ » ce qui se traduit par «Il vaut mieux en rire que d'en pleurer ». Cela veut dire que cette femme a choisi la mort plutôt que la vérité qui lui attirerait la honte... Profitons de cette morale pour en faire une autre : telle que l'histoire de la dot est racontée, d'aucuns se moqueront des Dagara. Mais dans les sociétés où il n'y a pas la dot, c'est même pire. Il y a des sociétés où le cocufié a droit de vie ou de mort sur le fautif....

Par des analyses, certaines significations des mythes ont été révélées. Ces significations peuvent permettre une compréhension mutuelle entre les partisans de la dot et leurs antagonistes si cette pratique est perçue comme des formes de vie conformément à Terres de sens de Fontanille et Couegnas (2018). Dans ces conditions, les significations de la dot peuvent influer concrètement sur des comportements allant dans le sens du développement, de l'épanouissement humain surtout si les interprétations suivantes sont prises en compte car elles sont plus approfondies que les précédentes. 


\subsubsection{Par la dot dagara la femme tient la vie entière de son époux et de la société}

A bien comprendre entre les lignes des mythes dagara, il apparait que par la dot la femme tient la vie entière de son époux car en cas d'adultère de la femme, c'est l'époux qui meurt et non la fautive. C'est on ne peut plus paradoxal certes. Mais c'est ce paradoxe qui est la pierre angulaire du système d'égalité entre l'homme et la femme dans la société dagara. La poire semble être partagée en deux parts égales pour raison d'égalité. D'une part l'époux peut prétendre à plus d'une femme. La femme, elle, ne peut rêver qu'à un seul époux. D'autre part l'épouse peut décider de la vie de son mari. Celui-ci, lui, en aucune façon, ne peut encore prétendre à un tel pouvoir... Et le tout, tient de la dot. Sans elle, l'homme n'est pas prémuni contre l'adultère. Sans elle, la femme ne peut rêver tenir la vie d'un homme. Sans elle la société entière sera tout sauf dagara. Le schéma suivant l'explique.

Schéma : Hiérarchie sociale dagara par la dot

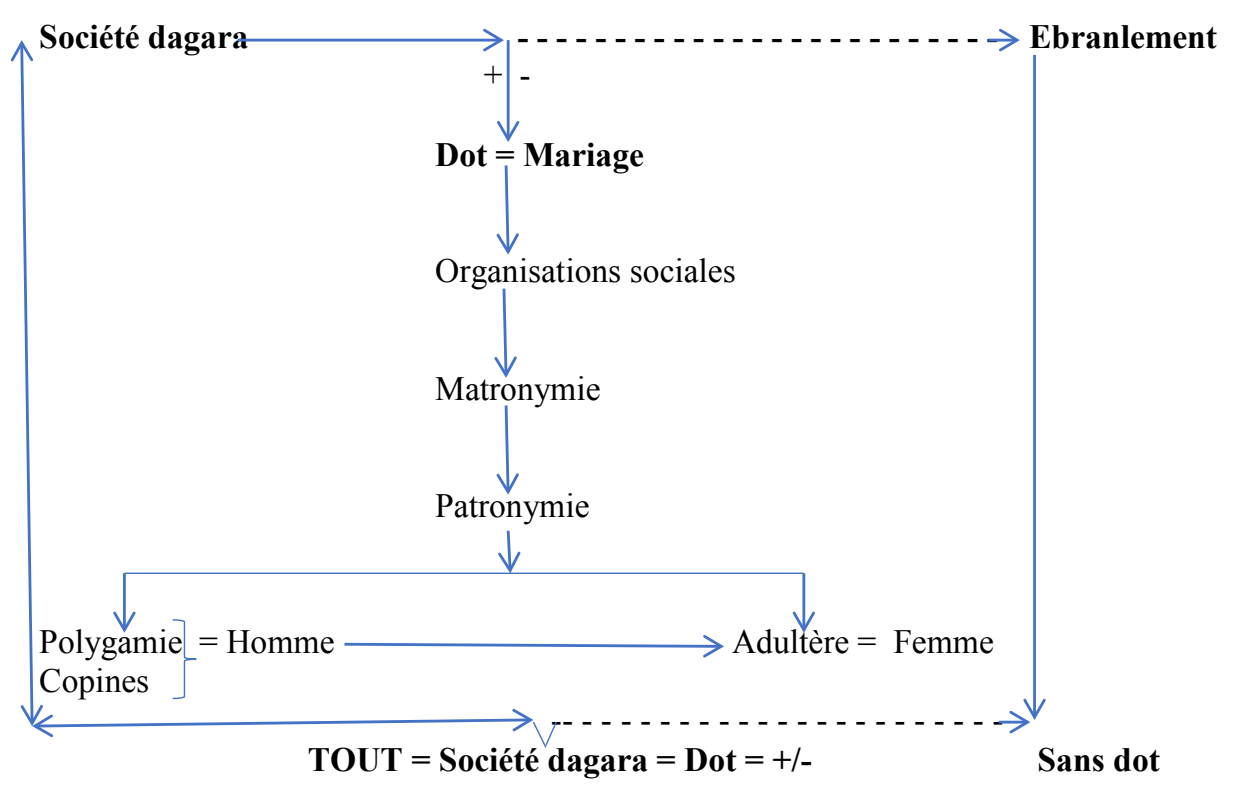

Comprendre ce schéma de la manière suivante : ce schéma peut se lire dans deux sens c'est-à-dire par le haut on peut aller vers la droite ou vers le bas qui est susceptible d'être aussi un point de départ par le TOUT, pour aller soit à droite soit à gauche. Ce sont les raisons pour lesquelles 
seuls les points de départs comportent des flèches à double sens. Dans tous les cas, lorsque l'on scrute très bien le schéma, le haut et le bas de celui-ci sont identiques et en caractères gras. Prenons le départ par le bas. Ce type de lecture est révélateur car il s'oriente vers une compréhension selon laquelle la société dagara est un TOUT. Et c'est la base, c'est le fondement de cette communauté burkinabè dont la pierre angulaire est la dot d'où le signe égal $(=)$ pour marquer la synonymie des trois termes: TOUT = Société dagara $=$ Dot.

Le départ pris peut s'orienter premièrement vers la droite : $c^{\prime}$ est un départ sans avenir. C'est le néant. C'est une tendance vers moins l'infini $(-\infty)$ matérialisé par le signe moins (-) et par les pointillés achevés par une flèche terminale parce qu'elle y prend fin. Mais elle indique qu'elle est une cause ; le manque de dot est une cause sociale dont la manifestation est l'ébranlement de TOUT d'où la synonymie suivante exprimée par : TOUT = Société dagara $=$ moins $(-)=$ Sans dot. Le gras de cette synonymie sur le schéma, marque sa gravité.

Par contre s'orienter deuxièmement vers la gauche c'est accepter la société dagara avec ses formes de vie telle la dot. C'est pourquoi cette flèche de gauche remonte vers la «Société dagara» qui suit un chemin à sens unique montré par la flèche unidirectionnelle qui est la continuité de celle du départ. A mi-chemin cette flèche peut, dans un premier temps, se poursuivre. C'est alors une suite sans avenir car elle est vouée au néant. Elle a une tendance vers moins l'infini $(-\infty)$ matérialisé par le signe moins (-) et par les pointillés achevés par le terme «Ebranlement ». Sur le schéma, le moins (-) est donc pour la flèche en pointillés.

Mais cette flèche n'est pas terminale car elle n'y prend pas fin. Elle indique la cause de cet «Ebranlement» par une flèche pleine pour exprimer la fermeté, la certitude que cette manifestation ou cette déstabilisation de la société dagara a une cause dénommée "Sans dot». On y assiste alors à la phase terminale de cette flèche négative unidirectionnelle. Elle ne remonte pas car la cause, la source ou les racines sont toujours vers le bas parfois cachées. Elle croise plutôt « Sans dot » qui est le lieu des horizons sans avenir. "Sans dot» a une tendance vers moins l'infini $(-\infty)$ d'où la synonymie suivante dont l'importance est exprimée sur le schéma par le gras : TOUT $=$ Société dagara $=$ moins $(-)=$ Sans dot.

A mi-chemin cette même flèche peut, dans un deuxième temps, descendre et aller vers le bas. C'est alors une suite positive marquée par le signe plus $(+)$ car elle est orientée vers la « Dot» qui signifie « Mariage». 
Cela est écrit sur le schéma $:$ Dot $=$ Mariage $=$ plus $(+)$. Cette synonymie est mise en gras pour traduire la gravité du lien entre la dot, le mariage et la positivité importante engendrée par ce rapport.

Le mariage positif par la dot engendre des «Organisations sociales ». C'est la flèche descendante qui exprime cette relation filiale. Celles-ci ne sont pas possibles sans le mariage avec dot. Ces «Organisations sociales» sont nombreuses, mais hiérarchisées. La première est la matronymie secondée par la patronymie. Une fois le mariage célébré conformément aux normes sociales, l'homme semble rattraper son rang second par la possibilité d'avoir plusieurs femmes, «Polygamie », et des «Copines » : c'est une des expressions égalitaires de la société dagara. L'«Homme» dagara même polygame peut avoir des copines d'où sur le schéma : Polygamie, Copines = Homme. La condition sine qua est : payer la dot.

La femme à plusieurs partenaires est frappée d'adultère : une faute très grave. Cela est matérialisé sur le schéma: Adultère $=$ Femme. Autrement dit l'adultère est un trait caractéristique féminin et non pas masculin. En revanche la femme a la force de tenir la vie de l'homme et de la société entière : c'est une autre des expressions égalitaires du Dagara. L'homme est dépourvu de cette force. Mais sans la dot la femme ne peut avoir ce pouvoir.

Après explication du schéma poursuivons nos idées : nous avons souvent évoqué l'égalité entre l'homme et la femme, mais une analyse plus poussée peut indiquer que l'ancêtre a favorisé la femme voire sa mère par rapport à l'homme voire son père ou lui-même si l'on pense que la polygamie, dans le passé, avait germé et jailli des esprits des hommes viriles. Mais rien n'indique qu'à cette époque, la polygamie ne pouvait pas être décidée par des femmes.

En effet, pas plus tard qu'hier seulement, certaines mamans clamaient encore à qui voulait bien les entendre que c'étaient elles qui recherchaient et sélectionnaient les copines pour leurs époux. C'étaient elles qui tenaient les bourses de leurs maris: par les petits commerces de dolo, galettes..., elles possédaient plus de revient que ceux-ci qui étaient interdits de vente de produits agricoles, pastoraux. Tout homme qui s'adonnait à de tels échanges, surtout dans l'optique d'aller courtiser des filles, était très mal perçu: on ne le comptait plus parmi les hommes valeureux de la société. Les hommes qui pratiquaient de tels mauvais commerces, passaient toujours par des femmes complices dépréciées. 
Rien n'indique qu'à cette époque, la polygamie ne pouvait pas être une décision des femmes. En effet, à cette époque, une femme sans coépouse ou dont l'époux n'avait pas de copine, était très mal appréciée. Des attributs lui étaient destinés tels : femme acariâtre, insociable, sorcière, voleuse de sauces, de to, de piment, de soumbala...

Dans la société dagara, toutes les preuves sont données qu'un homme véritablement viril ne peut se contenter d'une femme. Ces preuves tiennent de l'institution de la polygamie possible tirée des mythes présentés. Or les ancêtres d'alors ne prenaient jamais une telle décision pour complaire à un seul individu. C'était toujours pour le bien de toute la société. C'était aussi sur la base de moult expériences qu'ils avaient vécues.

La polygamie fut donc non seulement pour les travaux de tous ordres notamment champêtres mais surtout pour le bien-être psychique, l'équilibre mental dont le rêve de beaucoup d'enfants car à cette époque «mariez-vous et multipliez-vous » était encore la règle d'or...

Par la dot la femme tient l'homme et la société entière. Une analyse plus approfondie des mythes le confirme: lorsque dans les récits mythologiques donnés, le père disait à sa fille que c'était son mari qui mourrait en premier lieu, selon nous, c'était un véritable test d'amour de celle-ci pour son époux. Autrement dit si elle l'aimait réellement, si elle le voulait de tout son cœur, etc., elle ferait en sorte que son mari ne mourût pas !...

Tout donner à la femme par la dot faisait partie des grands secrets du couple dagara. De nos jours ce n'est plus secret pour personne, raison pour laquelle on peut en faire un objet de recherche scientifique voire divulguée, publique.... Mais il existe plus de raisons : l'institution dotale entière est même menacée de disparition par des administrations africaines voire burkinabè plus enclines aux mœurs d'ailleurs. Or elle a des valeurs certaines que nous prenons pour un bébé dans un bain. Alors il ne faut pas jeter le bébé avec l'eau du bain. Autre raison est que les hommes comme les femmes se prémunissent contre l'adultère : lire ci-dessous.

\subsubsection{Comme la femme ne se veut plus idéale...}

Il n'existe point de femme idéale : cette vérité, l'ancêtre dagara ne l'ignorait pas. Cependant il avait remis entre les mains de la femme la vie de l'homme et de la société entière par la dot. C'est par souci d'égalité par rapport à l'homme polygame, capable d'être entouré de copines... 
L'histoire $^{8}$ suivante qui n'est pas un mythe sur la dot mais véhiculée dans la société dagara, montre à quel point il n'est pas de femme idéale.

Il était une fois une femme qui souffrait énormément de cyphose, en Dagara "gore ». Et son époux en pâtissait autant. Il courait partout, faisait tout pour lui obtenir la guérison. Il vint à consulter un guérisseur qui lui dit ceci: "Je n'ai pas de médicament contre le mal de ta femme. Mais j'en connais un. Dis à ta femme d'être moins méchante, d'être gentille envers tout le monde surtout envers toi et elle guérira ». Le mari n'en croyait pas ses yeux. Il discuta très longuement avec le guérisseur pour persuader celui-ci afin qu'il lui donnât un remède à la hauteur du mal dont son épouse souffrait tant.

Le guérisseur finit par donner la potion magique et lui dit: « Tu feras quelque chose pour ton épouse. En revanche elle aussi fera quelque chose pour toi. Voici ta part: ce n'est pas toi qui es malade mais tu boiras cette décoction de patient en lieu et place de ta femme souffrante et devant elle. Ton épouse obtiendra alors la guérison. Quant à ta femme, dis-lui d'être gentille envers tout le monde surtout envers toi et elle guérira. En outre remets-lui cet œuf. Dis-lui qu'elle tient ainsi entre ses mains ta vie entière. Tant qu'elle sera gentille envers toi, tu seras heureux. Mais si elle casse cet œuf, tu mourras. »

Dès les premiers jours les prescriptions du guérisseur furent respectées à merveille. La femme fut guérie. Puis le temps passa mais non pas sans heurt. A chaque dispute la femme rappelait régulièrement à son époux de quoi elle était capable. Elle imposait respect envers elle. Quant au mari quand bien il avait raison il se dépêchait de reconnaître son tort et de demander pardon.

Mais un jour cette femme crut avoir assez de toujours accorder le pardon. Elle menaça son mari déjà à genoux et en supplications. Elle s'approcha de lui, le foudroya de regards fort furieux, le dépassa avec vitesse, entra dans la chambre, sortit l'œuf, le brandit et le fracassa contre le sol.

Aussitôt la femme redevint bossue. L'homme, quant à lui, n'eut aucun mal.

${ }^{8}$ Le vrai auteur de toutes ces histoires est la société dagara. Nous-même les connaissons mais par la recherche elles nous sont rappelées par nos informateurs comme Somda Kuu-yaa-san dit Gbèdaa et alii du village de Kognègan. 
Concluons: comme la femme ne se veut plus idéale, tous les hommes se protègent contre la mort par l'adultère. Le danger retombe toujours sur la femme infidèle. Comment? On retrouve là encore tout le secret; chasser le naturel, il revient au galop : lire infra. Des femmes aussi se prémunissent contre la mort par l'adultère. Lorsque c'est ainsi, les deux conjoints sont dits «blindés ». En Dagara on dit «B $\mathrm{B}$ za kpaw nâ » ou «B za bina to ». Alors chacun fait ce qu'il veut. Le couple n'est donc pas exemplaire. Les enfants de ces conjoints, leurs parents... tout le monde fait les frais de leurs comportements répréhensibles. En ville, même au village, on voit bien comment des Dagara souffrent de l'infidélité de leurs parents en couple. Mais il y a toujours un qui finit par l'emporter et c'est le désastre tant pour le conjoint vaincu que pour le dit vainqueur et pour toutes leurs relations sociales à commencer par les plus proches. Et la leçon à tirer est que toute société a besoin de règles de conduite : la dot est un de ces principes.

\subsubsection{Par la dot le vrai mariage dagara est « indissoluble »}

Le mariage dagara était d'abord social et ce n'était pas un jeu de mots. Ce n'était pas théorique mais pratique. Tout commençait par la difficulté à avoir une femme. Chaque famille voulait et en même temps ne voulait pas donner sa fille en mariage. Le départ d'une fille de sa famille était difficile à concevoir encore plus à accepter. Il était considéré comme une perte avec l'incertitude que les filles étrangères, en Dagara "Sâânbe » ou «Sââm $\varepsilon$ » que les fils de la famille marieront, ne fussent à la hauteur des leurs données aussi en mariage.

Tous les mythes étudiés montrent comment le Dagara est attaché à sa fille n'en déplaise à ceux qui voient par-là la manifestation du complexe d'CEdipe. La conclusion est: chaque famille étant consciente de cet attachement originel, elle fera en sorte que ce ne soit pas un conjoint qui, tout seul, décide de la conjonction ou de la dissolution de son mariage. Outre les stratagèmes évoqués supra pour résoudre les problèmes conjugaux, voici un autre stratège social que nous désignons par l'expression: la consolidation des foyers conjugaux par les bergers, en Dagara «Kob-tur-dicü-kobr-a-nuu» évoqués dans le récit de la dot dagara.

On fait expressément souffrir les dits bergers qui sont les pierres angulaires de la dot à remettre. Du même coup, aucun conjoint ne peut les outrepasser pour dissoudre le mariage qu'ils ont scellé de la sueur de leurs fronts. C'est pourquoi on dit que par la dot le vrai mariage dagara est 
" indissoluble» sans l'accord des bergers. Ce rôle social a une valeur telle qu'il fallait l'introduire et le traiter à part entière. L'indissolubilité du vrai mariage dagara est, en majeure partie, due au rôle social des bergers.

La dissolution du mariage n'est pas encouragée dans la société dagara. Elle est même combattue. C'est pourquoi elle est à l'origine des différences entre les grosses sommes d'argent et la panoplie d'animaux qui accompagnent les trois cent soixante (360) cauris. Ces sommes étaient les mêmes d'une femme dagara à l'autre. Mais l'histoire retient que des Dagara, parce que plus forts physiquement ou matériellement (plus riches), prenaient un mauvais plaisir à déposséder les autres époux de leurs femmes. Alors les coutumes dagara ont donné la possibilité aux hommes dits puissants de jouir de leurs forces mais d'en payer le prix. Depuis ce temps, c'est la famille de la femme qui fixe à loisir ces sommes souvent très élevées pour empêcher la dépossession.

Pour une raison ou une autre (mésentente, conflit...) l'amant peut être dans l'impossibilité de se rendre chez le cocu pour rembourser à celuici sa dot. C'est l'une des raisons pour lesquelles cet amant doit alors recourir à la famille d'origine de la femme. Dans ce cas, celle-ci va aussi être exigeante et avec raison. Non seulement la dépossession d'une femme n'est pas bénie par la société, mails surtout le nouvel amant lui demande un travail supplémentaire, pénible et plein de risque : elle peut écoper de tout acte de vengeance, de colère, de la part du cocufié.

La famille de la femme a alors le droit de majorer excessivement la dot. Une dette de ce genre se paye en une fois. Elle doit donc être intégrale. Elle ne souffre pas de négociation ni d'arrangement. Elle est teintée de colère, de vengeance, de tous mauvais penchants. La famille de la femme prélèvera d'abord sa part avant d'appeler le cocufié pour lui remettre sa dot qui doit être la même que celle qu'il avait donnée pour mériter sa femme. Cette dot ne souffrira d'aucun franc ou cauris de plus ni de moins par rapport à la première.

Si la femme en question avait engendré une fille chez le premier mari, la dot de celle-ci, à son mariage, serait identique à celle de sa mère. Si elle avait enfanté une autre fille chez le second mari, la dot de celle-ci, à son mariage, serait identique à celle de sa mère en prenant en compte la première majoration de la famille maternelle. Si un troisième mari survenait, une seconde majoration serait encore possible mais pas obligatoire. Autant de maris autant de hausses ou surestimations possibles. 
Voilà quelle est l'origine des différences des dots entre femmes. Mais ne pas oublier que certains éléments de la dot, les plus importants, sont figés, immuables.

Dans la société dagara il est fréquent d'entendre les expressions suivantes: "retirer la femme d'autrui est un signe de virilité, de bravoure" (Deblv nâa)... ou "seules les fées méritent autant de maris qu'elles désirent». Fée en Dagara se dit "Pow kâwele», ce qui veut dire "femme de beauté féerique». Ces expressions ne sont pas des vertus institutionnalisées par la société dagara. Elles sont des réalités, certes, mais surtout liées à des circonstances acceptées et validées occasionnellement par la société dagara connue pour ses bonnes mœurs, pour ses valeurs sociales.

Toutes les vertus maritales, tous les rôles collectifs de cette société en faveur du mariage, tiennent de la dot qui, chassée, reviendra au galop.

\subsubsection{Chasser le naturel, il revient au galop}

La dot et la polygamie sont surtout les deux aspects combattus et rejetés dans le mariage africain en général et dagara en particulier. Or par la dot et la polygamie de nombreux problèmes trouvaient leurs solutions. Sans la dot aucun homme célibataire ou marié en quête d'autres épouses, ne pouvait prétendre à une fille : aller chez elle ou la faire venir chez lui, la sortir librement, se pavaner avec elle, faire un enfant... Presque tout le monde avait un conjoint. L'infidélité était quasiment impossible car il n'y avait pas d'hôtel de passe. Or faire l'amour au dehors était une malédiction tant pour les fautifs que pour toute la société. Alors il fallait s'en abstenir !

C'est en adoptant des mœurs qui ne sont pas les leurs que des Africains ou des Dagara deviennent parfois les pires ennemis de la dot. C'est donc par ces mœurs d'ailleurs que des maux dangereux ont frappé et frappent toujours les Dagara. Ces maux sont: les hôtels de passe, l'infidélité, les hommes et femmes libres qui sont de véritables prédateurs de couples... Nombreux alors sont les Dagara qui finissent par reconnaître l'importance de la dot sans laquelle ces maux prendront toujours le dessus. C'est pourquoi l'on dit «Chasser le naturel, il revient au galop ».

\subsection{Perspectives : Solutions à une épreuve de vérité}

Certaines solutions sont déjà proposées par D. D. R. Kusiélé (1999: 62-65). En outre les perspectives en question sont des projections qui se veulent être aussi des remèdes aux réalités souffrantes relatives à la dot 
dagara. Ces souffrances sont souvent imputables aux mauvais comportements de certains Dagara qui se présentent alors comme les brebis galeuses. Autres causes sont les destinations ratées de la dot. Mais la suppression de celle-ci peut être une invite au pire qui viendra occuper la place dotale.

\subsubsection{Les arrangements avec l'administration}

L'opinion générale a fini par retenir que la loi burkinabè, notation à travers Le code des personnes et de la famille (CPF), (1989), est contre la dot en son «Article 244 » page 65. Si cela est vrai, d'autant que nul n'est censé ignoré la loi, nous aussi, nous ne sommes point contre la loi. Et nous y insistons. Cependant, au cas où certains Burkinabè l'ignoreraient aussi, il importe que ceux-ci sachent qu'un certain nombre d'arrangements ont été faits avec les villages dagara.

Au nombre des arrangements évoqués supra, on peut citer ceuxci sur la base des enquêtes auprès de certains de nos informateurs ${ }^{9}$ : Certaines parties de la dot, notamment celles financières, animales, ne sont plus exigibles en termes de bœufs, cauris... à un prétendant qui n'en a pas. Celui-ci peut donc s'acquitter de ce devoir coutumier à l'aide des différentes monnaies modernes en vigueur notamment le franc CFA. Certaines parties de la dot, notamment celles financières, animales, ont été homologuées. En conséquence le «Carv » de douze mille (12 000) cauris est réduit et équivaut à douze mille francs CFA (12000 F CFA), treize mille (13 000) cauris c'est treize mille Frances CFA (13 000f CFA), quatorze mille (14 000) cauris c'est quatorze mille Francs CFA (14000 F CFA), etc. Les deux bœufs sont revus à la baisse à quarante-cinq mille (45000) Francs CFA en raison de vingt-cinq mille (25 000) Francs CFA la génisse et vingt mille (20 000) Francs CFA le taurillon. A partir de ces calculs, le prix du troisième ou nième bœuf, selon qu'il est mâle ou femelle, peut être connu d'avance.

Un père qui tient à tuer un bœuf, en Dagara «pow-ya-naab »10, peut négocier avec son gendre car aucune des sommes proposées par

\footnotetext{
${ }_{9}^{9}$ Informateurs : Kpanyawnè Somda Kuu-yaa-san dit Gbèdaa et alii, département de Dissihn, village de Kognègan, le 23/03/2017.

10 «psw-ya-naab» : chez les Dagara, un père dont une des filles se marie, et reçoit les premiers animaux dotaux, doit prélever un qu'il offrira aux Ancêtres mais surtout c'est pour que la société entière se régale : c'est le «pow-ya-naab».
} 
l'administration ne permet aujourd'hui d'acquérir un bœuf si chétif soit-il. En dépit des propositions faites par l'administration burkinabè, certaines familles préfèrent toujours doter les femmes de leurs fils par les cauris notamment les trois cent soixante (360) cauris. Elles refusent de les monnayer pour les raisons suivantes : d'abord c'est un autre signe de fierté pour dire qu'il y en a ; cette famille ne manque pas de cauris.

Ensuite on pense que monnayer le moindre aspect de la dot est une troncation des coutumes dagara. Ipso facto cela peut avoir un impact négatif sur la femme. Pour le moins que l'on puisse dire : elle est «moins femme dagara » que les autres femmes dotées à la manière typiquement ancestrale ; elle est faible, fragile, sujette à tout défaut de femme. Puis l'on pense qu'il importe de minimiser les risques tels que l'inclination à dépenser très facilement le Franc CFA, à ne pas respecter le troc qui veut que cet argent demeure pour doter une autre femme...

Le Dagara ne voulait pas de cette «pécuniarisation» de la dot. Ces sentiments de réfutation de la "pécuniarisation» se focalisent en ceux exprimés par D. D. R. Kusiélé (1999 : 62). Mais selon notre entendement, ces arrangements sont des preuves d'ordre comportemental, c'est-à-dire plus pratique que théorique, indiquant que par rapport au bon changement, à l'évolution, le Dagara y est parfois plus enclin que l'on ne le croit. Mais l'ancêtre dagara avait même prévu des arrangements avant ceux faits avec l'administration.

\subsubsection{L'arrangement ancestral génial}

En poussant davantage les analyses dotales, il nous est apparu que l'ancêtre dagara avait déjà prévu des arrangements destinés à faciliter la dot. Les accommodements suivants indiquent à quel point il était prévoyant, avisé et donc génial.

Premièrement la hiérarchisation des constituants de la dot: le premier élément et le plus important est petit, quasiment insignifiant. C'est le «Libir kpëë », les trois cent soixante (360) cauris. Ce chiffre, si chétif soitil, suffit pour posséder une femme. Le Dagara, lui-même, le dit le plus souvent: "Cââpile, $\varepsilon$ vle nı a kpëë». Cela se traduit par: petit mais plus important. En cas d'arrangement la dot ne commence pas par le gros mais par le petit aspect «Cââpile ». L'arrangement ancestral génial est donc dans le petit, le quasi insignifiant.

Rares sont les Dagara qui ne s'enorgueillissent pas de l'insignifiance de leur dot ancestrale. Ils s'en vantent à commencer par 
ceux qui ont l'expérience de dots onéreuses d'autres ethnies. Alors ils se proposent souvent de venir en aide à leurs amis ou aux hommes d'autres ethnies qui courtisent les filles dagara mais prêts à se déresponsabiliser par peur de cette dot. Et qui dit peur dit souvent ignorance. L'aide tantôt évoquée consiste alors à bien expliquer la dot pour qu'elle soit comprise et acceptée.

Par expérience nous avons connu des couples qui se sont contentés de ces trois cent soixante (360) cauris pendant de très longues années avant de remettre le reste de la dot. Le secret est: il faut que ce soit sincère et respectueux des règles de l'art.

Deuxièmement le reste de la dot, le gros lot, se fait aussi par arrangement surtout si les difficultés sont connues. Très souvent, c'est au niveau du troisième ou énième bœuf que les véritables problèmes se posent. Habituellement voici comment cela se passe : à chaque réclamation de ce bœuf, il existe une "phrase fétiche expiatoire» de tout danger qu'engendrerait cette dette: "C'est la dette de l'orphelin ». En Dagara : «Bikpieb-sân- $\tilde{v} »$.

Si un bœuf de ce genre n'avait jamais été remis dans la lignée maternelle de la mariée, il faut le réclamer, mais ne jamais le prendre sous peine de sanction très grave des ancêtres maternels. Ici, tout pouvoir est à la lignée maternelle dont les vivants peuvent déjà porter plainte pour éviter tout désagrément.

Si un bœuf de ce genre, de par le passé, avait été remis dans la lignée maternelle de la mariée, il faut le réclamer sans outrepasser la "phrase fétiche expiatoire » dont, par opposition, useraient les endettés : "C'est la dette de l'orphelin ». Même si un bœuf de ce genre doit se remettre réellement, cela se fait habituellement lorsqu'une des filles de cette femme dotée se marie.

Troisièmement la dot se remettait étape par étape. Cela pouvait prendre des années voire "pendant plus de 15 ans », écrit D. Dèr. R. Kusiélé (1999: 63). Mais comme l'ancêtre dagara avait permis les arrangements, cela fait que de nos jours, toutes les étapes peuvent se dérouler en même temps. C'est surtout le cas lorsque le gendre ou la belle-famille vit très loin. Citons notamment les cas des «Nı-bolı». Ce terme se traduit par « Dagara allophones » ou «non-Dagara » qui viennent très souvent de contrées très lointaines. N'oublions surtout pas de citer les nombreux fonctionnaires même dagara aux comportements «nt-bolı» soit parce qu'ils habitent 
effectivement loin soit parce qu'ils allèguent les contraintes de leurs fonctions, etc.

En somme l'arrangement ancestral et génial de l'ensemble de la dot se trouve à plusieurs niveaux : le petit ou le quasi insignifiant, la sincérité, le respect des règles de l'art, la «phrase fétiche expiatoire», les «nonDagara », les Dagara fonctionnaires... Tous ces niveaux sont réductibles en un seul : le respect des règles de l'art.

En dépit de ces arrangements prévus de manière ingénieuse par les ancêtres pour faciliter l'acquittement de dot, on note des brebis galeuses dans la société dagara.

\subsubsection{Les brebis galeuses}

L'expression, "les brebis galeuses », désigne certains Dagara qui se sont laissé prendre par tous les vices de ce monde. En Dagara on les appelle les «Nı folı». En français familier africain voire burkinabè ce sont des «faux-types». Sur le plan littéraire ce sont des «hommes sans valeur». Parmi ceux-ci on en trouve qui pensent que la dot est une occasion en or pour vendre leurs filles ou pour "s'enrichir». Voilà une des raisons fondamentales pour lesquelles des filles dagara rejettent catégoriquement la dot. C'est du «mercantilisme»:

"Le mercantilisme: C'est faire de la dot ce que nous avons rejeté tout à l'heure: le "prix de la fiancée ». Il y a sans doute des parents (individus ou groupes) qui font de la femme un objet à vendre, et du mariage une occasion de s'enrichir ». La dot est alors manipulée en dehors des normes fixées par la coutume elle-même» (D. D. R. Kusiélé, 1999 : 62).

\subsubsection{Destination de la dot}

La dot est destinée à doter la femme d'un fils de la famille. La dot ne se dépense dans aucun autre cadre que la dot, le mariage. Elle ne sert pas à faire des dépenses futiles tels l'habillement, boisson, construction de maison d'habitation, etc. Cependant il n'existe pas d'interdit mortel en la matière au point que d'aucuns y voient la porte ouverte à la dilapidation. Nombreux sont ceux qui ne respectent pas strictement ces règles établies depuis la nuit des temps par les ancêtres et qui, du jour au lendemain, se retrouvent face à une dot dont ils ne peuvent s'acquitter.

Toujours au sujet des manières de se servir de la dot: si la génisse vêle et fait prospérer le parc animalier familial, on peut en profiter. On 
peut prélever certaines têtes d'animaux pour vendre et résoudre des problèmes estimés nécessaires.

En somme, il faut comprendre qu'en dépit de certains caractères rigides et même très contraignants de la dot, les coutumes, voire les ancêtres, laissent une très grande part d'ingéniosité à l'acteur dotal. C'est à lui d'en profiter ou d'y perdre. On peut dire que c'est grâce à cette acclimatation, voire à ce type d'aggiornamento, que la dot a franchi la nuit des temps pour être toujours d'actualité.

\subsubsection{La nature a horreur du vide}

Nous proposons de ne pas rejeter nos mœurs. Si nous renions les nôtres notamment la dot, d'autres coutumes prendront place en nous car la nature a horreur du vide. Alors les filles dagara, voire africaines, doteront tôt ou tard leurs gendres si cela n'est déjà pas une réalité dans des familles où les filles «souffrent visiblement» pour avoir des maris. En effet on constate de plus en plus des villas érigées, des cadeaux de grande valeur destinés aux prétendants ou aux futurs gendres sous le couvert de présent de mariage au nouveau couple...

\section{Conclusion}

Notre terrain de recherche n'a pas été le territoire français encore moins l'environnement rural du Limousin qui a permis à J. Fontanille et à N. Couegnas (2018) de mener leur démarche sémiotique par leur œuvre Terres de sens qui est en réalité une méthode plus pratique que théorique. Notre terrain est africain, burkinabè. Il est plus précisément dagara. A l'image du milieu du Limousin notre espace de recherche est aussi rural et il véhicule des imaginaires, des mythes dont nous avons fait notre corpus.

Nous nous sommes appesanti sur le récit de la dot dagara que nos informateurs nous ont narré oralement mais aussi sur nos expériences personnelles d'autant que nous sommes natif du milieu dont les coutumes font souvent objet de nos recherches scientifiques. Des études antérieures notamment celles de J. Fontanille et N. Couegnas (2018) nous ont servi de modèle pour toutes nos idées allant dans le sens des formes de vie, de l'imaginaire, des récits, des mythes.

Nous l'avons fait à bon escient. En effet, c'est grâce aux idées émanant de ces prédécesseurs que nous avons pu comprendre et révéler ceci : il faut toujours chercher à comprendre la signification de la dot 
dagara, voire de toute institution dotale, avant toute intention de rejet et ce, à l'intérieur d'une forme de vie et d'un mode d'identification. En effet la dot dagara est une forme de vie que nous avons détaillée et qui peut permettre d'éviter les conflits de valeurs et de mœurs.

Ces antagonismes sont souvent sources de fléaux sociaux à la manière de l'évêque missionnaire brésilien dévoré avec tout son équipage (J. Fontanille, N. Couegnas, 2018 : 39). Par expérience nous savons que des Burkinabè occupant des fonctions administratives importantes ont été victimes de ces conflits sociaux pour avoir combattu cette dot dagara que nous avons interprétée à l'aide des idées de J. Paré (1998) et de P. Ricœur (1986).

En lieu et place de la coopérative proposée par Fontanille et $\mathrm{N}$. Couegnas (2018) dans Terres de sens, nous retenons l'action collective pour ne pas mourir. Nous insistons davantage sur cette action collective qui est récurrente dans toutes nos recherches mais dont la base est d'abord individuelle. Autrement dit, pour un bon développement ou épanouissement collectif il importe que sur la base des idées ou écrits des chercheurs, les lecteurs se laissent influencer en adoptant réellement des comportements positifs d'abord individuels qui sont les preuves de convictions personnelles. La part collective aura pour base ces états de faits individuels et sera plus sure, plus efficace et prompte.

En agissant ainsi nous pensons alors que le problème posé au début de nos réflexions est résolu. Il l'est par la vérification des hypothèses suivantes : la dot dagara est bien connue ; elle est clairement expliquée et interprétée pour les partis antagonistes. Notre objectif est atteint d'autant que cette pratique sociale qui est la dot est mieux comprise et acceptée par les deux mondes opposés. Les résultats obtenus sont visibles à travers la dot dagara qui est maintenant connue, interprétée et acceptée de part et d'autre. L'adhésion se manifeste à travers les comportements, les écrits comme le nôtre, les discours, les lois administratives...

\section{Bibliographie}

\section{Informateurs les plus reguliers}

1. BEKUONE, Kambou I-i-mwin dit Saakoum du village de Navrikpè

2. KPANYAWNE, Somda Kuu-yaa-san dit Gbèdaa, représentant du groupe, village de Kognègan

3. KPANYAWNE, Somda Mathias du village de Kognègan 
4. KPANYAWNE, Somé Guillaume dit Gaoussou du village de Kognègan

5. BEKUONE, sœur Somé KPièri Domè Brigitte (1994), Enquête sur les alliances dans la culture africaine notamment au pays dagari

6. Burkina Faso, 16/11/1989, Code des Personnes et de la famille (CPF), Zatu an VII.

7. DIOP, Cheikh Anta (1982), L'unité culturelle de l'Afrique noire : Domaine du patriarcat et du matriarcat dans l'antiquité classique, Paris, Présence Africaine, [1959 pour la 1ère édition].

8. FONTANILLE, Jacques (2015), Formes de vie, Liège, Presses Universitaires de Liège, Collection Sigilla, 278 p. ISBN : 978-2-87562066-8.

9. FONTANILLE, Jacques, COUEGNAS, Nicolas (2018), Terres de sens : Essai d'anthroposémiotique, Presses Universitaires de Limoges (PULIM), 276 p.

10. GBAANE, Dabiré Constantin (1978), Bu-nyèri ni bu-bè-nyèri (LE VISIBLE ET L'INVISIBLE) : Une interprétation de la mentalité animiste en guise de contribution à l'entreprise philosophique universelle, Koumi

11. GBAANE, Dabiré Constantin (1983), Nisaal : L'homme comme relation, Tome I, Ecole des gradués de l'Université Laval, Faculté de Philosophie, Thèse.

12. KUSIELE, Dabiré Dèr Raphaël (1999), La dot et son rôle dans le mariage traditionnel, Ouagadougou, 9e Grande conférence du Ministère de la communication et de la culture, $67 \mathrm{p}$.

13. PARE, Joseph (1998), "Pour une sémiotique à base herméneutique » in Annales de l'Université de Ouagadougou, Série A, Volume X, p.p. 7790.

14. RICOEUR, Paul (1986), Du texte à l'action: Essais d'herméneutique II, Paris, Editions du Seuil.

\section{WIKIPEDIA}

1. Récupéré et revisité le 04/05/2021 de Le Grand Robert de la langue française, Le Robert / SEJER, 2005, www.lerobert.com, version électronique 2.0

2. Récupéré et revisité le 04/05/2021 du Microsoft ${ }^{\circledR}$ Encarta ${ }^{\circledR}$ 2009. (C) 1993-2008 Microsoft Corporation. 